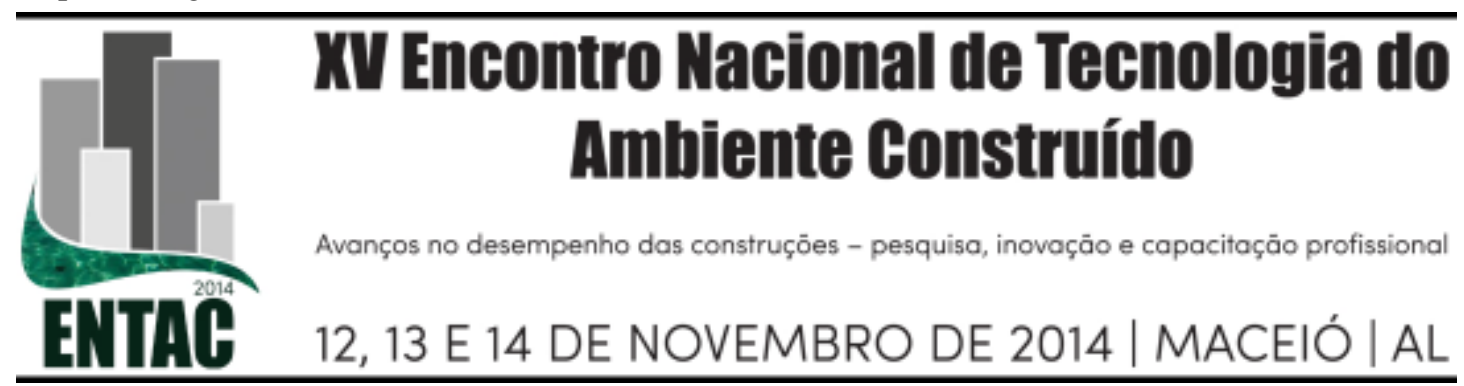

\title{
ESTUDO DE CASO EM UM LABORATÓRIO UNIVERSITÁRIO PAULISTA COM UMA ABORDAGEM SUSTENTÁVEL
}

\author{
MICELI, CAROLINA PARDO (1); FONTANINI, PATRICIA STELLA \\ PUCHARELLI (2); JACINTO, ANA ELIZABETE P.G.A. (3); PIMENTEL, LIA \\ LORENA(4); LINTZ, ROSA CRISTINA CECCHE (5)
}

(1) Pontifícia Universidade Católica de Campinas, e-mail: carol_pardo_miceli@yahoo.com.br, (2) Pontifícia Universidade Católica de Campinas, e-mail: pspucha@terra.com.br, (3) Pontifícia Universidade Católica de Campinas, e-mail: anajacinto@gmail.com, (4) Pontifícia Universidade Católica de Campinas, e-mail: lialp@puc-campinas.edu.br, (5) Universidade Estadual de Campinas, e-mail: rosacclintz@ft.unicamp.br

\begin{abstract}
RESUMO
O desenvolvimento do setor da construção tem impulsionado a busca de novas tecnologias para proporcionar a otimização dos sistemas envolvidos no trabalho civil. A maioria das falhas em sistemas de construção se origina nos processos de concepção e execução. A correta definição de requisitos do sistema é essencial, para o sucesso do projeto sustentável. Existem alguns requisitos gerais na definição dos sistemas de construção que necessitam ser considerados, tais como: padrão de qualidade, custo, economia de insumos, nível tecnológico, durabilidade, segurança, facilidade de operação e manutenção. $\mathrm{Na}$ presente pesquisa é realizada uma pesquisa bibliográfica e, a identificação da aplicabilidade de conceitos de zoneamento bioclimático no desempenho em edifícios, para torná-los mais sustentáveis. Como estudo de caso foi analisado o edifício dos laboratórios de solos da Pontifícia Universidade Católica de Campinas (PUC-Campinas), localizada em Campinas - SP. Identificaram-se adequações na edificação com o uso das normas da ABNT-NBR. Por fim, foram sugeridas medidas a serem tomadas para adequação ambiental, garantindo uma reforma da edificação em conformidade com os critérios de sustentabilidade mencionados pela Norma.
\end{abstract}

Palavras-chave: Conforto Térmico, Zoneamento Bioclimático, Sustentabilidade

\begin{abstract}
The construction sector development has studied new technologies to increase the systems optimization involved in work. Most failures in buildings system originate from the design processes and their implementation. The correct definition of requirement systems is essential to sustainable project. There are some general requirements in the system Project building are needed, such as: quality pattern, target costing, economics input, technological level, durability, safety, operation and maintenance. This research was started with the bibliographical research and identification of the bioclimatic zoning concepts that apply in buildings. It was shown how to make them more sustainable. As a case study, it is analyzed the soil laboratory of the Pontificia Universidade Católica de Campinas (PUC), located in Campinas-SP. Some possibilities are identified with the standards ABNT use. Finally, they are suggested measures to be taken a more sustainable building, ensuring the edification rebuilding in accordance with sustainability criteria.
\end{abstract}

Keywords: Thermal Comfort, Bioclimatic Zone, Sustainability 


\section{INTRODUÇÃO}

Pesquisas relatam que o Brasil possui um grande déficit habitacional, que pode ser representado por um número próximo de 7,0 milhões de habitações. Essa estatística reflete a necessidade urgente moradias. Existe ainda uma parcela das moradias existentes que ainda carecem de uma readequação das condições mínimas necessárias.

A necessidade constante de construção de novas moradias não deve diminuir o cuidado na fase de projeto das residências. Atualmente, ao se investigar as construções existentes, observa-se que uma grande porcentagem das edificações foram realizadas de maneira inapropriada, tanto em relação à qualidade de vida, quanto com o meioambiente. $\mathrm{O}$ acelerado crescimento da sociedade, impacta exigências ambientais, que cada vez mais estão entrelaçadas nas leis orgânicas municipais e estaduais. Pinto et al., 2009, destaca os conceitos de sustentabilidade, que podem ser apresentados em três pilares: social, econômico e ambiental, e tem como objetivo diminuir o impacto ambiental, e favorecer o desenvolvimento de projetos mais integrados em seu ambiente, dessa forma, garantindo a qualidade de vida dos moradores das habitações.

O artigo apresenta o conteúdo de uma pesquisa desenvolvida na Pontifícia Universidade Católica de Campinas, que propõe a avaliação prévia do conforto térmico do edifício que abriga o laboratório de mecânica dos solos. A avaliação inicial para a proposição de modificações e adaptações sustentáveis, que nem sempre exigem um aporte de recursos financeiros para a adequação do espaço já existente. A pesquisa ilustra a aplicação de conhecimentos, para a adaptação do edifício já construído que apresenta problemas de conforto térmico, adicionados a avaliação de conceitos de sustentabilidade, para garantir uma melhor adequação, a partir de medidas simples de adaptação. A pesquisa mostra resultados da análise da Norma, proposta de adequação a partir de condições sustentáveis, e com um dos resultados a minimização do consumo de energia. Mostra também alguns cálculos do desempenho térmico conforme a Norma de zoneamento bioclimático. O trabalho é desenvolvido de forma exploratória e preliminar em um estudo de iniciação científica PUC-Campinas. O objetivo do estudo de caso é verificar a possibilidade de propor melhorias no projeto para a adaptação de uma edificação.

\section{REVISÃO BIBLIOGRÁFICA}

\subsection{Sustentável}

Atualmente, o projeto sustentável é um novo modelo de projeto de edificação, que já tem sua própria história - e o projeto sustentável resulta de uma evolução do projeto arquitetônico. Pode-se também dizer que uma edificação sustentável também é uma edificação inteligente, a medida que a mesma busca atender os requisitos do cliente, com o mínimo gasto de recursos possíveis. E ainda os projetos sustentáveis tentam atender às necessidades ambientais do futuro empreendimento, de forma que os recursos sejam aproveitados de maneira eficiente ao consumo de energia, água e materiais, sem comprometer os recursos das gerações futuras. Lins (2012) define a sustentabilidade corporativa, e explica o que é projeto sustentável:

"Sustentabilidade corporativa nada mais é do que, a incorporação de critérios socioambientais no processo de tomada de decisões, conferindo a eles peso semelhante ao das variáveis econômicofinanceiras. Em outras palavras, significa dizer que em decisões de investimentos, compra e venda de ativos, mudanças no processo produtivo ou desenvolvimento de novos produtos serão levados em conta aspectos como emissão de gases de efeito estufa, existência de 
passivos ambientais, respeito aos direitos humanos na cadeia produtiva ou pagamento pelo uso da água, dentre outros" (LINS,2012).

\subsection{Edifícios Sustentáveis}

O conceito de edifícios sustentáveis intercepta tanto os conceitos de edifícios inteligentes, como os princípios discutidos no Lean Construction, onde existe a preocupação com a redução dos desperdícios encontrados durante o processo de projeto, construção e manutenção das edificações. Para Godfaurd et. al. (2004), existe uma importância singular em compreender e participar dos processos de criação do ambiente construído. Nessa linha de pensamento é possível buscar inspiração da natureza, não só sobre os materiais e mecanismos, mas sobre o risco, e manutenção de um projeto executado. O mesmo autor explica que a construção sustentável deve considerar todo o ciclo de vida dos edifícios, que buscam a qualidade ambiental, funcionalidade e valores futuros das novas gerações.

Para Godfaurd et. al. (2004) a construção sustentável é, portanto, a pensativa integração da arquitetura, com todos os aspectos dos projetos, de forma integrada (projetos: elétricos, mecânicos, estruturais). Além da preocupação estética tradicional de aglomeração, orientação, escala proporcional, textura, sombra e luz, a equipe de design precisa se preocupar com custos de longo prazo: ambiental, econômico e humano. Os edifícios sustentáveis contribuem para o aumento de qualidade de vida dos habitantes.

Segundo Martinez (2009) estão sendo implantados os conceitos de sustentabilidade, na construção civil, com o intuito de reduzir o consumo de energia (desperdícios inerentes que o conceito do Lean Construction aborda), como é o caso das construções sustentáveis, conhecidas por sua denominação em língua inglesa, como Green Building.

Martinez (2009) ainda menciona que os edifícios ou construções verdes são concebidos dentro do conceito de que as edificações agridam o mínimo possível o meio ambiente. $\mathrm{O}$ conceito envolve desde a escolha dos materiais que serão utilizados durante a construção, até os custos ambientais e de manutenção do edifício ao longo de sua vida útil.

\subsection{Edifícios inteligentes}

Segundo Messias (2007), o conceito de edifícios sustentáveis tem evoluído muito, e alguns estudos apontam que o mesmo caminha próximo aos conceitos de otimização e inteligência em edificações. Os Edifícios inteligentes, assim como sugere a definição, seguindo o IBI (Intelligent Buildings Institute dos EUA), são os edifícios que oferecem um ambiente produtivo e econômico, a partir da otimização de quatro elementos básicos: estrutura (componentes estruturais do edifício, elementos de arquitetura, acabamentos de interiores e moveis), sistemas (controle de ambiente, calefação, ventilação, ar-condicionado, luz, segurança e energia elétrica), serviços (comunicação de voz, dados, imagens, limpeza) e gerenciamento (ferramentas para controlar o edifício) das inter-relações entre eles.

"Uma edificação "inteligente" deve promover aos usuários conforto, segurança e sobre tudo economia, tanto em custos diretos (água, luz, telefone, etc.), quanto em custos indiretos, tais como manutenção e operação, além de possuir na sua concepção todos os estudos de questões ambientais, sustentabilidade, aproveitamento de recursos e de tecnologias de forma eficiente" (MESSIAS, 2007). 
Os Edifícios inteligentes apresentam, atualmente, uma tendência para um custo compatível com o grande mercado de consumo, desde que planejado em projeto preliminar e projeto integrado.

\subsection{Conforto ambiental}

Segundo Pinto et al. (2009), o conceito de sustentabilidade ambiental implica em diminuir o impacto ambiental que uma construção causa em seu ambiente, porém garantindo a qualidade de vida daqueles que nela residem ou a freqüentam. Para isso, um dos fatores mais importantes é a redução do consumo de energia, a qual é um recurso escasso mal utilizado nas edificações. Apesar da população brasileira ter um baixo consumo - se comparado com outros países - ainda há potencial para a redução do mesmo segundo a NBR 15220 - Desempenho Térmico de Edificações, que propõe uma avaliação de conforto térmico, esta podendo ser feita tanto na fase de projeto, ou após a sua realização. Em relação à edificação construída, a avaliação deve ser realizada a partir de medições "in loco" de variáveis representativas ao desempenho (NBR 15575), enquanto que na fase de projeto esta avaliação pode ser feita por meio de simulação computacional ou a partir da verificação do cumprimento de diretrizes construtivas. A NBR 15220 - parte III apresenta recomendações quanto ao desempenho térmico de habitações, e recomendações de diretrizes construtivas e detalhamento de estratégias de condicionamento térmico passivo, com base em parâmetros e condições de contornos fixados. Outros parâmetros que também poderiam ser adotados com relação a eficiência energética seria o Procel (RTQ-C, 2014), entretanto, não contemplado nessa pesquisa.

\section{METODOLOGIA}

Para o desenvolvimento do trabalho foi realizada uma pesquisa bibliográfica para se compreender a sustentabilidade e importantes conceitos de conforto térmico aplicados ao tema. Escolheu-se analisar o conforto térmico, devido a facilidade em se coletar as informações, realizar o diagnóstico e promover sugestões de melhorias a partir de conceitos de sustentabilidade em projetos. Foi estudado então o zoneamento bioclimático da região de Campinas relativos a edificação, e realizados os cálculos de desempenho térmico. Esse laboratório tem a função de acolher os estudantes nas aulas experimentais ligadas a área de solos (Mecânica dos Solos e Geologia) na Pontifícia Universidade Católica de Campinas (PUC-Campinas). A metodologia contemplou as seguintes etapas, (i) Conhecimento sobre as normas vigentes para o aprofundamento técnico em zoneamento bioclimático (ii) Definição de Parâmetros necessários ao projeto; (iii) Cálculos de desempenho térmico e Zoneamento bioclimático. Para atender ao item (ii) e (iii) foram estudadas as diretrizes construtivas de forma a atender ao Zoneamento bioclimático da cidade de Campinas, onde se encontra o laboratório, são:

A cidade de Campinas-SP se encontra na Zona Bioclimática Brasileira 3, de acordo com a NBR 15220 - Desempenho térmico de edificações. E esta caracterizada por ser uma zona de aquecimento solar da edificação, esta norma estabelece para a zona 3 para as seguintes diretrizes construtivas:

- Aberturas para ventilação de tamanho médio e sombreamento das aberturas permitindo sol durante o inverno;

- Vedações externas Leves e refletoras nas paredes e Leves e isoladas nas coberturas;

- Estratégias de condicionamento térmico passivo para o Verão: Ventilação cruzada; e para o Inverno: Aquecimento solar da edificação e 
Vedações internas pesadas (inércia térmica).

Os parâmetros de Desempenho Térmico especificados para a Zona Bioclimática de Campinas são:

- Transmitância Térmica $<$ ou $=3,60$

- Atraso Térmico $<$ ou $=4,30$

- Fator Solar $<$ ou $=4,00$

\section{ESTUDO DE CASO}

Após o estudo de conceitos de sustentabilidade e das Normas de Conforto Térmico, optou-se por estudar uma edificação que, atualmente, tem como finalidade o desenvolvimento de atividades didáticas para as classes de Engenharia Civil e Ambiental com o conteúdo de Mecânica dos Solos e Geologia. O laboratório faz parte do complexo do CEATEC, e fica localizado no Campus I da Pontifícia Universidade Católica de Campinas. O laboratório abriga três ambientes distintos: uma sala de aula com seis bancadas para desenvolvimento de experimento, uma sala de aula anfiteatro para o desenvolvimento de aulas expositivas e um mezanino onde existem salas menores para docentes da Faculdade com dedicação exclusiva. Além disso, o espaço conta com vários equipamentos, e amostras de rochas e solos. As dimensões do laboratório analisado: 11,75 metros x 13,20 metros, sendo que, a envoltória da edificação é composta por vários materiais (alvenaria, portas e janelas) cujas áreas são apresentadas na tabela 1 .

Tabela 1 - Áreas que compõe a envoltória da edificação

\begin{tabular}{|c|c|c|}
\hline Materiais & Dimensões & $\begin{array}{c}\text { Total em metros } \\
\text { quadrados }\end{array}$ \\
\hline Bloco de Cimento & & $281,69 \mathrm{~m}^{2}$ \\
\hline Porta de Madeira & $2,10 \mathrm{~m} \times 0,90 \mathrm{~m}$ & $1,90 \mathrm{~m}^{2}$ \\
\hline Portão de Ferro & $3,30 \mathrm{~m} \times 3,40 \mathrm{~m}$ & $11,22 \mathrm{~m}^{2}$ \\
\hline Janelas de Vidro & $(1,50 \times 13,20) \mathrm{m}+(2,10 \times 13,20) \mathrm{m}$ & $47,52 \mathrm{~m}^{2}$ \\
\hline
\end{tabular}

A Tabela 2 mostra os cálculos de Fator Solar para o Laboratório e nas figuras 1 a 4 apresentam vistas internas e da fachada da edificação.

Tabela 2 - Fator Solar do Laboratório

\begin{tabular}{|c|c|c|c|c|}
\hline & \multicolumn{4}{|c|}{ Laboratório de Solos } \\
\hline & $\mathbf{m}^{\mathbf{2}}$ & $\mathbf{\%} \mathbf{~ m}^{\mathbf{2}}$ & $\mathbf{F S}$ & Ponderado \\
\hline Bloco & 281,60 & 0,82 & 6,18 & 5,08 \\
\hline Vidro & 47,52 & 0,14 & 14,60 & 2,03 \\
\hline Madeira & 1,90 & 0,01 & 0,30 & 0,00 \\
\hline Ferro & 11,22 & 0,03 & 8,76 & 0,29 \\
\hline & 342,24 & & & \\
\hline & & & Fator Solar & 7,40 \\
\hline
\end{tabular}




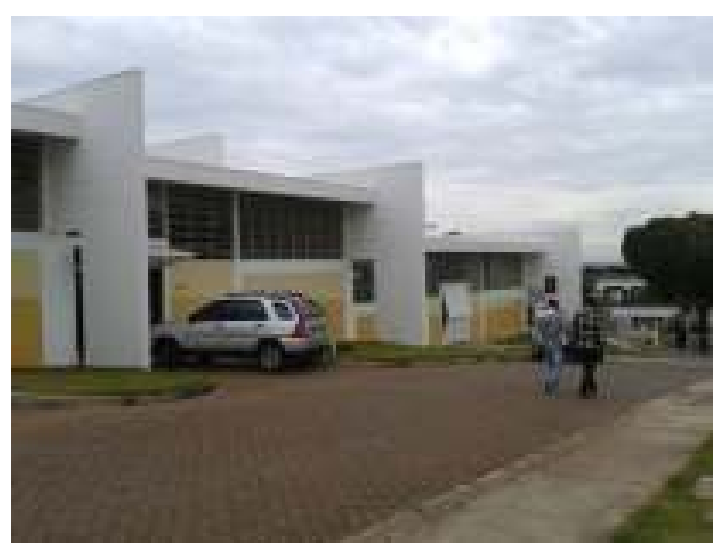

Figura 1 - Fachada do laboratório

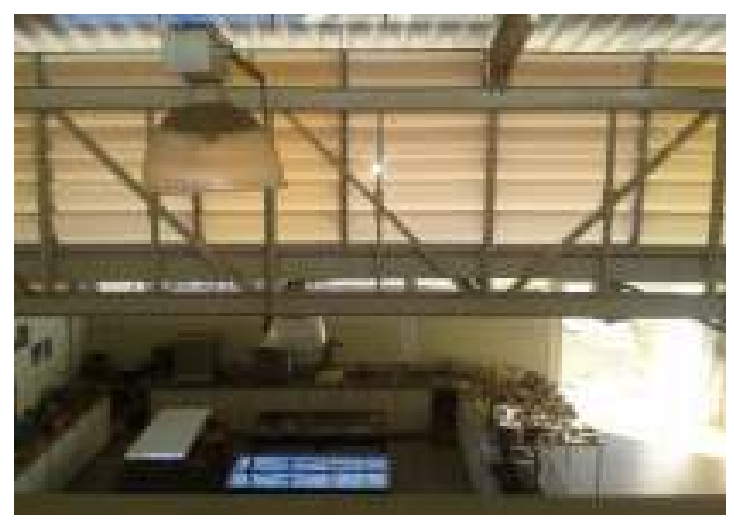

Figura 3 - Vista do Mezanino

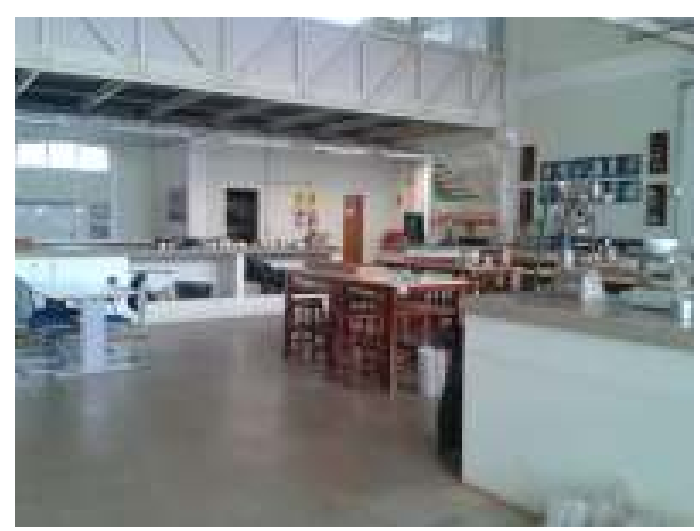

Figura 2 - Vista Interna

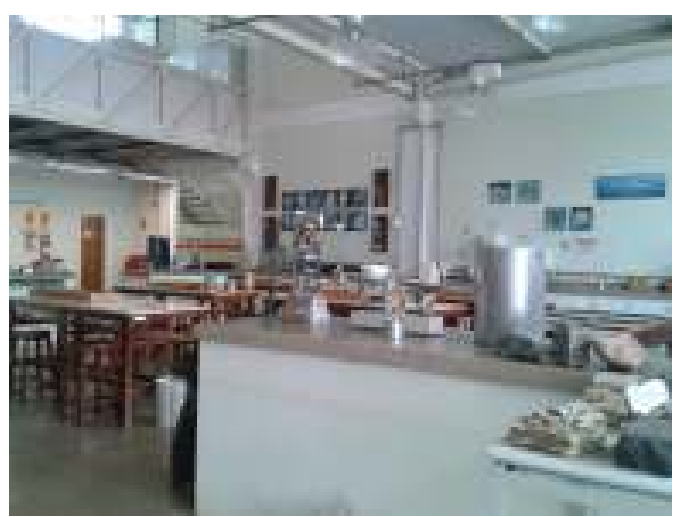

Figura 4 - Vista das Bancadas

\section{ANÁLISE DE RESULTADOS}

Os cálculos de desempenho térmico foram realizados para analisar a envoltória da edificação do laboratório em questão. Verificou-se a resistência térmica, resistência térmica total, transmitância térmica e capacidade térmica. Considerou-se a seguinte orientação solar: o sol da manhã cobre a fachada principal no período das 9:00 às 12:00hs, e começa a se por ao fundo da edificação por volta de 16:00hs (durante os meses de Setembro à Janeiro). A fachada norte recebe o sol da tarde, portanto, elevando a temperatura da edificação na parte de trás da edificação, principalmente após às 15:00hs, nas estações de verão e primavera a temperaturas altas (mais de 32 graus Celsius).

Observou-se que o Fator Solar (FS) da edificação (calculado) não atende ao previsto pela NBR 15220, para o zoneamento bioclimático da região de Campinas. O fator solar deveria ser igual ou inferior a 4,0. A envoltória analisada não atende a norma, e nem possibilita um bom conforto térmico. Para se obter os valores adequados a zona bioclimática foi refeito os cálculos do Fator Solar, a partir de novas especificações de projeto, tais como:

- Mudança para pintura clara externa (para cor Branca) $\rightarrow$ alpha $=0,20$

- Redução na metragem quadrada de pano de vidro. 


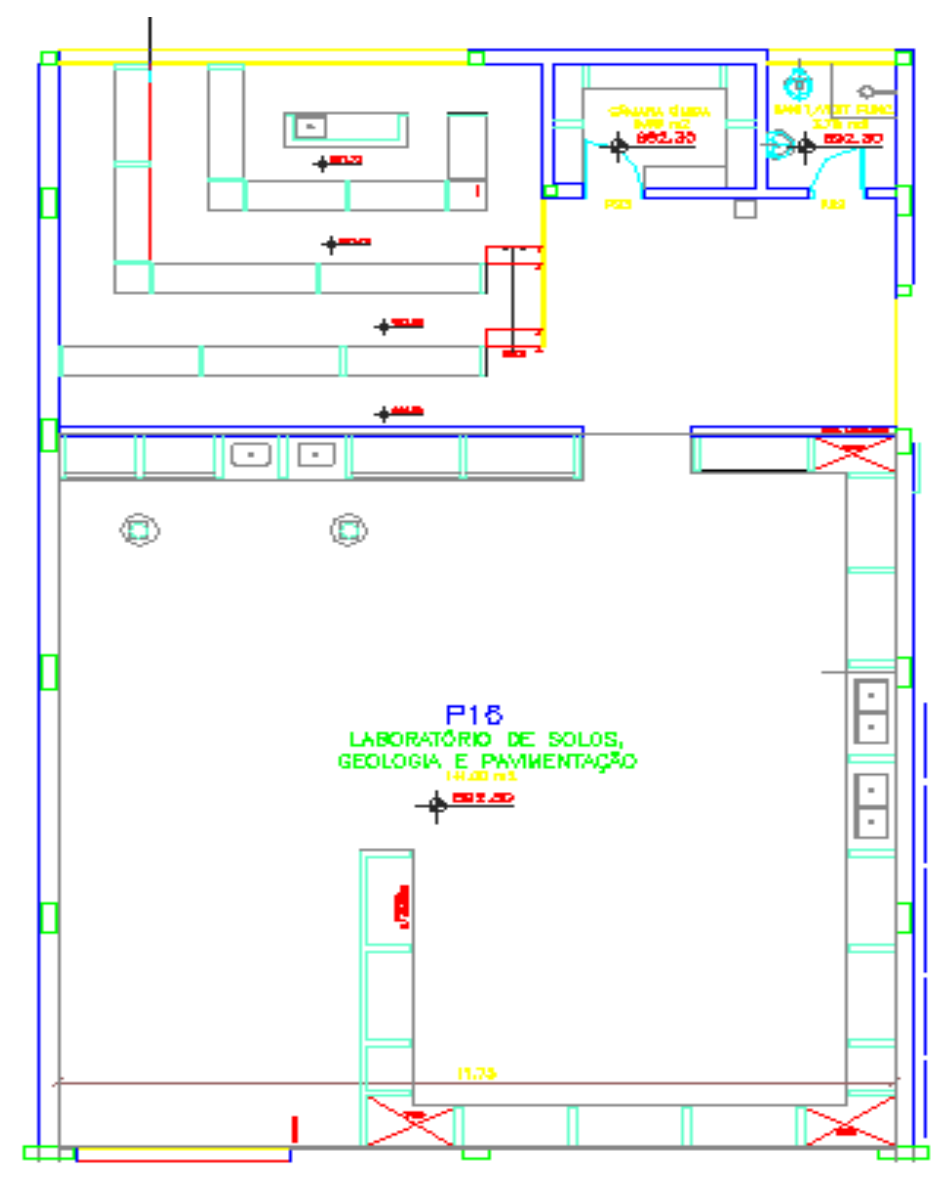

Figura 5 - Planta do Laboratório

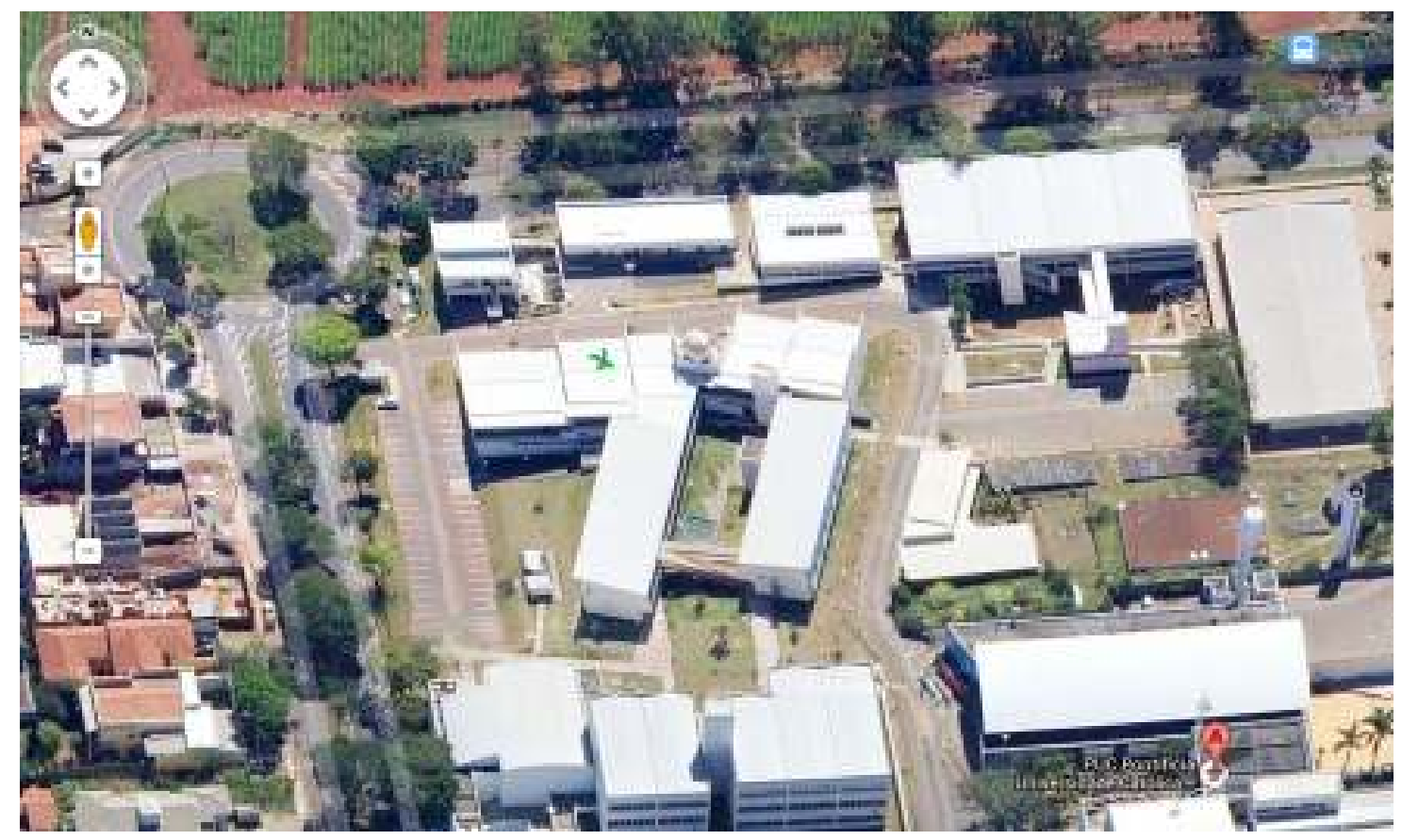

Figura 6 - Laboratório indicado com um " $X$ " verde, seguindo a indicação do Norte. Fonte: Google Earth (2014) 
Tabela 3 - Áreas contribuintes e Fator Solar

\begin{tabular}{|c|c|c|}
\hline Material Construtivo & Área contribuinte (\%) & Fator Solar (FS) \\
\hline Bloco de Concreto & $82,00 \%$ & 5,06 \\
\hline Madeira & $1,00 \%$ & 0,30 \\
\hline Ferro & $3,20 \%$ & 0,27 \\
\hline Vidro & $14,00 \%$ & 1,40 \\
\hline TOTAL & & 7,03 \\
\hline
\end{tabular}

Tabela 4 - Tabela com as sugestões de projeto

\begin{tabular}{|c|c|c|l|}
\hline CENÁRIOS & $\begin{array}{c}\text { Fator Solar } \\
\text { (FS) }\end{array}$ & $\begin{array}{c}\text { Percepção do } \\
\text { Conforto }\end{array}$ & Melhorias Sustentáveis sugeridas \\
\hline Cenário Atual & $\mathrm{FS}=7,03$ & $\begin{array}{c}\text { Conforto térmico } \\
\text { comprometido e } \\
\text { Iluminação } \\
\text { Insuficiente }\end{array}$ & \\
\hline Cenário 1 & $\mathrm{FS}=5,1$ & $\begin{array}{c}\text { Conforto térmico } \\
\text { com potencial de } \\
\text { melhoria na } \\
\text { temperatura do } \\
\text { ambiente }\end{array}$ & - Alteração da pintura externa para branco. \\
& FS Redução de $10 \mathrm{~m}^{2}$ de janelas de vidro. \\
\hline Cenário 2 & - & $\begin{array}{c}\text { Grande melhoria no } \\
\text { conforto térmico e na } \\
\text { Iluminação das salas } \\
\text { de aula }\end{array}$ & $\begin{array}{l}\text { - Alteração da pintura externa para branco, } \\
\text { - Redução de 15 } \mathrm{m}^{2} \text { de janelas de vidro e } \\
\text { sombreamento de 10 m² de janelas na } \\
\text { parte do mezanino. Redistribuição das } \\
\text { janelas restantes pela edificação. }\end{array}$ \\
\hline
\end{tabular}

\section{CONSIDERAÇÕES FINAIS}

As considerações apresentadas nos dois cenários tiveram o intuito de buscar a melhoria de qualidade e conforto das atividades desenvolvidas no laboratório de solos. A ventilação cruzada também foi apontada como uma solução viável, entretanto deveria estar previsto um novo posicionamento das aberturas, atualmente existentes na edificação. Além de atender as diretrizes construtivas especificadas para a zona bioclimática 3, a redução do pano de vidro e, a modificação da pintura nas paredes já possibilitaria uma melhora significativa no conforto. Concluí-se que para tornar a edificação sustentável, além de conhecer as técnicas disponíveis para a adequação da edificação, é necessária uma avaliação da edificação, com relação a sua adequação perante as normas técnicas disponíveis, para uma possível solução ambiental. A pesquisa apontou a importância da avaliação das edificações já construídas, e a possibilidade de facilmente indicar mudanças, que promovam o conforto e a sustentabilidade do ambiente. Deve-se enfatizar que, o conceito de conforto ambiental, corretamente aplicado em todas as obras e ainda contemple outras tecnologias, visa a redução do consumo de energia. Para atender, manter e preservar um ambiente 
agradável a todos é necessário corrigir as falhas, que agravam os problemas ambientais e minimizar o impacto com tecnologias viáveis adequadas a cada ambiente.

\section{AGRADECIMENTOS}

A PROPESQ - PRÓ-REITORIA DE PESQUISA DA PONTIFÍCIA UNIVERSIDADE CATÓLICA DE CAMPINA pela bolsa FAPIC de iniciação científica, e à Fundação de Amparo à Pesquisa do Estado de São Paulo (FAPESP), processo n ${ }^{\circ}$ 2014/16362-1, pelo apoio na divulgação da pesquisa.

\section{REFERÊNCIAS}

ASSOCIAÇÃO BRASILEIRA DE NORMAS TÉCNICAS (ABNT). NBR 15220: Desempenho térmico de edificações: CB-02. Setembro 2003. Parte 1. 7p.

ASSOCIAÇÃO BRASILEIRA DE NORMAS TÉCNICAS (ABNT). NBR 15220: Desempenho térmico de edificações: CB-02. Setembro 2003. Parte 2. 21p.

ASSOCIAÇÃO BRASILEIRA DE NORMAS TÉCNICAS (ABNT). NBR 15220: Desempenho térmico de edificações: CB-02. Setembro 2003. Parte 3. 24p.

ASSOCIAÇÃO BRASILEIRA DE NORMAS TÉCNICAS (ABNT). NBR 15572: Ensaios não destrutivos - Termografia - Guia para inspeção de equipamentos elétricos e mecânicos

BRASIL. Portaria Inmetro $\mathbf{n}^{\mathbf{0}}$ 372, de 17 de setembro de 2010. Regulamento Técnico da Qualidade para Eficiência Energética de Edifícios Comerciais, de Serviços e Públicos (RTQ-C). Disponível em: < http://www.inmetro.gov.br/legislacao/rtac/pdf/RTAC002100.pdf > Acesso em: JUL/2014.

GODFAURD, J.; CLEMENTS-CROOME, D.; JERONIMIDIS, G. Sustainable building solutions: a review of lessons from the natural world. 2004. 320 - 328f. The University of Reading, UK.

GOOGLE EARTH-MAPS. Disponível em: <http://mapas.google.com>. Acesso em: $\mathrm{AGO} / 2014$.

HILGENBERG, F.B. Sistemas de certificação ambiental para edifícios estudo de caso: Aqua. 2010. 149f. Dissertação (Pós-Graduação) - Universidade Federal do Paraná, Curitiba.

LINS, CLARISSA. Sustentabilidade Corporativa: desafios - Clipping - O Globo: Economia. 2011. 67f. In: Fundação Brasileira para o Desenvolvimento Sustentável. II Encontro Em Boa Companhia, Rio de Janeiro, São Conrado.

MARTINEZ, M. F. B. Avaliação Energética visando a certificação verde. 2009. $126 f$. Dissertação - (Mestre) - Universidade Federal do Rio Grande do Sul, Porto Alegre, 2009.

MESSIAS, ALAN FERNANDES. Edifícios "Inteligentes": A domótica aplicada à realidade brasileira. 2007. 43f. Monografia - Universidade Federal de Ouro Preto, Ouro Preto.

NEVES, R. P. A. Tecnologias utilizadas nos edifícios inteligentes. 2002. 144f. Dissertação (Mestre) - Universidade de São Paulo, São Carlos, 2002.

PINTO, L.V.C.; RODRIGUES, H.C. PIMENTEL, L.L.; LINTZ, R.C.C.L. M. J. A. Habitação de interesse social sustentável em argamassa armada. In: ELECS 2009 - V Encontro Nacional e III Encontro Latino-Americano sobre Edificações e Comunidades Sustentáveis, 2009.

RTQ-C - Disponível em: <HTTP:// http://www.procelinfo.com.br/>. Acessado em 17/08/2014.

UNITED STATES ENVIRONMENTAL PROTECTION AGENCY. Water Management Plan Revision 1, Region 1. ANO 2010. 15f. Plano de Gerenciamento - USA, EPA. 